\title{
Quantitative-Qualitative Data Acquisition Using a Benchtop Orbitrap Mass Spectrometer
}

\author{
Kevin P. Bateman, ${ }^{\text {a }}$ Markus Kellmann, ${ }^{\mathrm{b}}$ Helmut Muenster, ${ }^{\mathrm{b}}$ \\ Robert Papp, ${ }^{\mathrm{a}}$ and Lester Taylor ${ }^{\mathrm{c}}$ \\ a Merck Frosst Canada Ltd., Kirkland, Quebec, Canada \\ $\mathrm{b}$ Thermo Fisher Scientific, Bremen, Germany \\ c Thermo Fisher Scientific, San Jose, California, USA
}

Current approaches to discovery-stage drug metabolism studies (pharmacokinetics, microsomal stability, etc.) typically use triple-quadrupole-based approaches for quantitative analysis. This necessitates the optimization of parameters such as Q1 and Q3 $\mathrm{m} / \mathrm{z}$ values, collision energy, and interface voltages. These studies detect only the specified compound and information about other components, such as metabolites, is lost. The ability to perform full-scan acquisition for quantitative analysis would eliminate the need for compound optimization while enabling the detection of metabolites and other non-drug-related endogenous components. Such an instrument would have to provide sensitivity, selectivity, dynamic range, and scan speed suitable for discovery-stage quantitative studies. In this study, a prototype benchtop Orbitrap-based mass analyzer was used to collect both quantitative and qualitative data from human microsomal incubation samples as well as rat plasma from pharmacokinetic studies. Instrumental parameters such as scan speed, resolution, and mass accuracy are discussed in relation to the requirements for a quantitativequalitative workflow. The ability to perform highly selective quantitative analysis while simultaneously characterizing metabolites from both in vitro and in vivo studies is discussed. (J Am Soc Mass Spectrom 2009, 20, 1441-1450) (C) 2009 Published by Elsevier Inc. on behalf of American Society for Mass Spectrometry

$\mathrm{T}$ The widespread adoption of triple-quadrupole mass spectrometers for quantitative analysis in the pharmaceutical industry has been a major contributing factor to the reduced attrition ascribed to poor pharmacokinetic properties [1, 2]. The high degree of specificity provided by selected reaction monitoring (SRM) is a recognized strength of triple-quadrupolebased instruments, but it is also a limitation. Several investigators have described the potential advantages of using full-scan-based acquisition for quantitative analysis using a variety of mass analyzers [3-8]. High among these advantages is the ability to acquire information without the need for specific methods to be developed beforehand. For example, information about drug metabolites can be mined from the data post-acquisition, leading to so-called quantitative-qualitative workflows $[9,10]$. Additionally, information about nondrug components can also be investigated, whether to check for interferences or as a readout to study endogenous biological processes.

Many quantitative workflows in early drug discovery are focused on improving analytical throughput using fast liquid chromatography-mass spectrometry (LC-MS) approaches to screen large collections of compounds [11-13]. A significant bottleneck in these work-

Address reprint requests to Dr. Kevin P. Bateman, Merck Frosst Canada, Inc., 16711 Trans Canada Highway, P.O. Box 1005, Kirkland, QC, Canada H9H 3L1. E-mail: kevin_bateman@merck.com flows is the time needed to develop the SRM methods required for triple-quadrupole-based analysis. In cases where compounds do not fragment favorably (either non-specifically or not at all) the analyst is left with limited options, especially when throughput is a key requirement. The use of full-scan acquisition has the potential to provide a solution to these problems.

For a full-scan approach to be acceptable in a discovery environment it needs to provide sufficient selectivity, sensitivity, duty cycle, and dynamic range to ensure that analysis is not compromised. In other words, the benefits of ease of use and qualitative data acquisition are not as important as having appropriate quantitative capabilities. It is for these reasons that previous attempts to use quantitative-qualitative workflows have not been widely adopted. For example, full-scan analysis using a time-of-flight (TOF) mass analyzer lack sufficient sensitivity and dynamic range [4]. Recent improvements in TOF-based mass spectrometers hold promise for true quantitative-qualitative workflows to be developed [10].

A recently developed mass analyzer-the Orbitraphas many of the features required for a full-scan quantitative instrument [14]. It provides high mass resolution and mass accuracy, wide dynamic range, good duty cycle, and good sensitivity. The first commercial form of this instrument was a hybrid with a linear ion trap coupled with the Orbitrap. Recently, a nonhybrid 
version of the Orbitrap mass analyzer was commercialized with improved performance relative to the firstgeneration instrument [15]. This instrument is reduced in both size and cost, relative to the first-generation system, making it more accessible for use in routine drug discovery screening applications. The system is capable of generating fragmentation information in a nonselective manner using a collision cell without precursor ion selection. In this way structural information can be obtained on compounds of interest and fragment ions can be used for confirmation in targeted analyses.

This report describes the performance of this new benchtop Orbitrap mass spectrometer when applied to specific applications (microsomal stability and plasma drug level measurement) in a drug discovery environment. For the stability assay, relative quantitation of the parent compound was used and metabolite information was extracted post-acquisition. For plasma level measurements, absolute quantitation of drug compound using a within-run validation approach was used. The full-scan data were mined for circulating metabolites in plasma. The importance of chromatographic and mass resolution for selectivity is discussed. In addition, the availability of metabolite data from early discovery assays and its potential impact on program timelines are highlighted.

\section{Experimental}

\section{Reagents and Materials}

The synthesis of the cathepsin K inhibitors L-006235 and L-873724 has been previously reported [16, 17]. All solvents were LC-MS grade or better. Formic acid and acetonitrile were from Fisher Scientific (Ottawa, ON, Canada) and LC-MS grade water was purchased from VWR International (Quebec, QC, Canada). Columns were purchased from Thermo Fisher Scientific (Mississauga, ON, Canada) and analytical standards (verapamil, labatelol, urapidil, loperamide, nefazadone, buspirone, and haloperidol) were from Sigma. The analytical standards were dissolved in dimethylsulfoxide and combined into a synthetic mixture containing $10 \mu \mathrm{M}$ of each compound diluted in acetonitrile:water.

\section{Animals}

All procedures were approved by the Animal Care Committee at the Merck Frosst Center for Therapeutic Research according to the guidelines established by the Canadian Council on Animal Care. Sprague-Dawley rats $(n=3)$ with an average mass of $300 \mathrm{~g}$ were used in these studies.

\section{Microsomal Incubations}

Incubations with rat liver microsomes were conducted at $37^{\circ} \mathrm{C}$ with $0.25 \mathrm{mg} / \mathrm{mL}$ of microsomal protein in 0.1 $\mathrm{M}$ phosphate buffer, at a compound concentration of 1 $\mu \mathrm{M}$, in the presence of $1 \mathrm{mM}$ nicotinamide adenine dinucleotide phosphate (NADPH) oxidase and $1 \mathrm{mM}$ uridine 5'-diphosphoglucuronic acid (UDPGA) as cofactors and $2 \mathrm{mM} \mathrm{MgCl} 2$ in a total volume of $500 \mu \mathrm{L}$. Aliquots were taken at multiple time points $(5,10,15,30$, and $60 \mathrm{~min}$ ) quenched with 2 volumes of cold acetonitrile containing labetalol (200 nM) as internal standard. Samples were centrifuged for $15 \mathrm{~min}$ at $3000 \mathrm{~g}$ and the supernatant was transferred to high-performance liquid chromatography (HPLC) vials for LC-MS analysis.

\section{Plasma Collection and Preparation}

Following the oral dosing of L-006235 or L-873724 (10 $\mathrm{mg} / \mathrm{kg}$, dose volume of $10 \mathrm{~mL} / \mathrm{kg}$ with $1 \%$ methocel as vehicle, $n=3$ rats), blood samples were collected at $0.25,0.5,1,2,4$, and $6 \mathrm{~h}$ postdosing in addition to a predose sample. Whole blood $(400 \mu \mathrm{L})$ was collected into heparinized tubes and centrifuged (10 min, 1000g) to separate plasma and red blood cells. Plasma $(50 \mu \mathrm{L})$ was transferred into Eppendorf tubes quenched with acetonitrile $(150 \mu \mathrm{L})$ containing labetalol $(200 \mathrm{nM})$ as internal standard and vortexed briefly to precipitate the plasma proteins. Tubes were centrifuged at $4000 \mathrm{rpm}$ for $10 \mathrm{~min}$ to pelletize proteins and $120 \mu \mathrm{L}$ of supernatant was transferred to HPLC vials before analysis.

\section{Instrument Layout Overview}

The instrument used was a beta version of the Exactive $^{\mathrm{TM}}$ benchtop Orbitrap mass spectrometer. Samples were introduced into the API source by either direct infusion or a UHPLC system (Accela ${ }^{\mathrm{TM}}$ ). The source is similar to the commercial source of a TSQ Quantum Ultra $^{\mathrm{TM}}$. Ions are transferred from the source through four stages of differential pumping using radiofrequency (RF)-only multipoles into a curved RF-only trapping quadrupole (the C-trap). In the C-trap ions are accumulated and their energy is dampened using a bath gas (nitrogen). Ions are then injected through three further stages of differential pumping using a curved lens system into the Orbitrap analyzer, where mass spectra are acquired via image current detection. The vacuum inside the Orbitrap mass analyzer is maintained below 1e-09 mbar.

\section{Automatic Gain Control (AGC)}

Automatic control of the number of ions in the Orbitrap is performed by measuring the total ion charge using a prescan and by calculating the ion injection time for the analytical scan from this value. For very high mass scan rates, the previous analytical scan is used as a prescan to optimize the scan cycle time without compromising automatic gain control. Ion gating is performed using a fast split-lens setup that ensures the precise determination of the ion injection time. 


\section{High-Energy Collision Dissociation (HCD)}

The incorporation of an additional collision cell allows HCD (higher energy collision decomposition) experiments to be performed. Ions are passed through the C-trap into a multipole collision cell where they are fragmented and stored. Subsequently, the HCD cell voltages are ramped and ions are transferred back into the C-trap from which they are injected into the Orbitrap for detection.

\section{LC-MS Description}

Initially the mass spectrometer was calibrated by infusion of a standard test mixture of caffeine, MRFA tetrapeptide, and Ultramark. All subsequent mass analysis was done using this initial mass calibration and no internal calibration was used. Generic LC-MS conditions were used throughout with no optimization of source parameters or gradient conditions. Separations were performed by injecting $25 \mu \mathrm{L}$ of sample onto a $2.1 \times 100-m m$ Thermo Hypersil Gold C18 column with 1.9 micron particles. The chromatography was developed using a linear gradient from 2 to $95 \%$ acetonitrile versus $0.1 \%$ formic acid in $5 \mathrm{~min}$, at a flow rate of 500 $\mu \mathrm{L} / \mathrm{min}$. Standard MS source conditions compatible with the flow rate were used (capillary temperature, $290^{\circ} \mathrm{C}$; sheath flow, 50; spray voltage, $3000 \mathrm{~V}$; auxiliary temperature, $400^{\circ} \mathrm{C}$ ). Scan speed was set depending on the experiment and is described in more detail in the following text. The collection of HCD data used a fixed energy setting of $35 \mathrm{~V}$.

\section{Results and Discussion}

\section{Instrument Performance}

To determine instrument performance characteristics in terms of scan speed, resolution, and mass accuracy, a synthetic mixture of drug molecules was prepared and analyzed. The mixture was separated using the generic LC conditions described earlier. In one experiment the mass analyzer was set to acquire data at $10 \mathrm{~Hz}$, the fastest setting for the instrument. The resultant data are shown in Figure 1a. The individual scans are represented as dots on the chromatogram, indicating that for peaks that are $3-4 \mathrm{~s}$ wide $30-40$ points are obtained across the peak. Extracted mass spectra are shown as insets in Figure 1a. These spectra were taken from the peak top and peak base, revealing that the mass accuracy and resolution are preserved across the full dynamic range of the chromatographic peak. There-
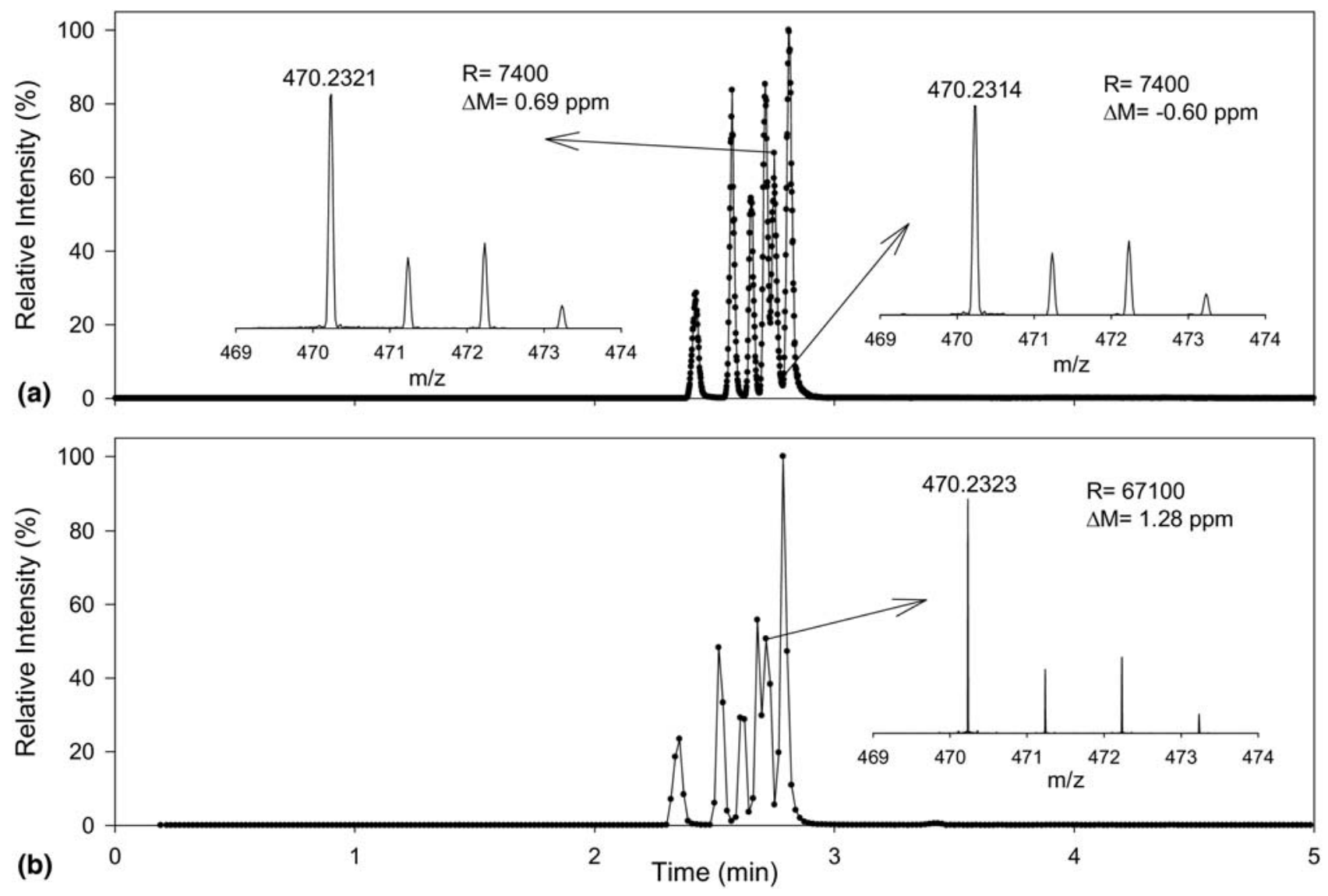

Figure 1. Comparison of (a) $10 \mathrm{~Hz}$ and (b) $1 \mathrm{~Hz}$ data acquisition rates using the Exactive ${ }^{\mathrm{TM}}$. Insets in (a) shows mass spectra taken from peak top and peak tail. Inset in (b) shows spectra taken from peak top. 
fore, whether the analysis involves either a low abundant ion or a high abundant ion, mass accuracy and mass resolution are maintained-this is important for accurate quantification in full-scan mode.

A similar experiment was repeated using a scan speed of $1 \mathrm{~Hz}$, with the resulting data shown in Figure 1b. In this case fewer points (black dots) are obtained across the chromatographic peak. However, the mass resolution is greatly improved, as shown by the mass spectrum in Figure 1b. The mass accuracy for the instrument was found to be less than 3 ppm when using external calibration. The data shown here indicate that, for this mass analyzer, speed is obtained as a concession to mass resolving power. Selectivity for specific analytes in complex matrices is obtained through mass resolution when using full-scan instruments, so it is important to understand the dependence of this parameter on scan speed when designing experiments.

Another important aspect of this instrument is the capability to collect fragment ion information without the need to select the precursor ion. Figure 2 shows data collected when two scan functions are used for acquisition, versus a single-function approach shown above. This approach is similar to the high-low switching used on QToF instruments for similar analysis [18]. In the first scan, full MS data are acquired at $10 \mathrm{~Hz}$ by sending the ions from the C-trap into the Orbitrap for mass analysis (Figure 2a and b). In the second scan, the ions are sent from the C-trap into the HCD cell, where they are fragmented and then sent back to the C-trap and, subsequently, to the Orbitrap for mass analysis (Figure $2 \mathrm{c}$ and $\mathrm{d}$ ). By switching back and forth between these two scans, two chromatograms are collected, one containing the total ion count (TIC) for nonfragmented ions (Figure 2a) and one for the fragmented ions (Figure 2c). Qualitatively these two chromatograms are almost identical. However, when one inspects the mass spectra extracted from the chromatographic peaks the differences are apparent. In Figure $2 b$ the mass spectrum from the peak indicated by the arrow in Figure $2 a$ is shown. The main feature of the spectrum is the $[\mathrm{M}+$ $\mathrm{H}]^{+}$for verapamil. The corresponding spectrum (Figure 2d) taken from the peak indicated in Figure 2c shows a rich fragment ion pattern for verapamil. These data are collected with both high resolution and high mass accuracy across the entire mass range and without loss of the low mass ions because this fragmentation occurs in the HCD cell and not in an ion trap. Although the
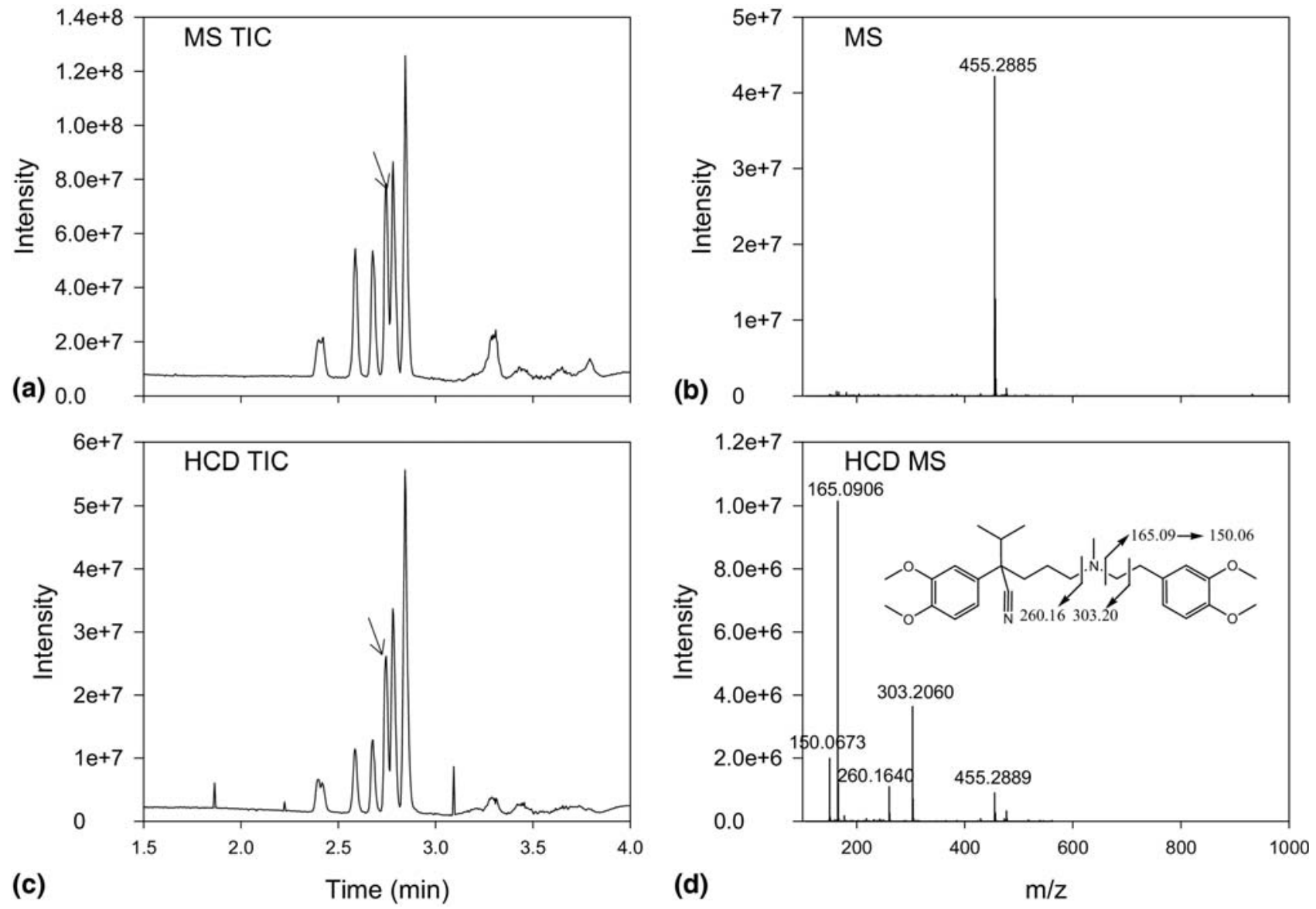

Figure 2. (a) MS and (c) HCD chromatograms for the analysis of a mixture of 6 compounds. (b) Mass spectrum extracted from peak labeled with arrow in (a) showing intact $[\mathrm{M}+\mathrm{H}]^{+}$for verapamil. (d) HCD mass spectrum from peak labeled with arrow in (c). Structure in (d) shows assignment of fragment ions for verapamil. 
absolute intensity of the fragment ion spectrum has decreased, the sum of the ion intensity in Figure $2 \mathrm{~d}$ indicates that minimal losses occur by using HCD. This is in contrast to the large scattering losses typically seen when using in-source fragmentation to do similar type experiments on single-stage mass analyzers. Because this fragmentation is generated without precursor ion selection, the ability to link fragment ions to a particular precursor ion will depend on chromatographic resolu- tion or software algorithms for peak deconvolution or both.

\section{Microsomal Stability Samples}

Analysis of microsomal stability samples is typically performed on triple-quadrupole mass spectrometers to have sufficient robustness, selectivity, and sensitivity. Quantitation is done relative to a time-zero sample. This
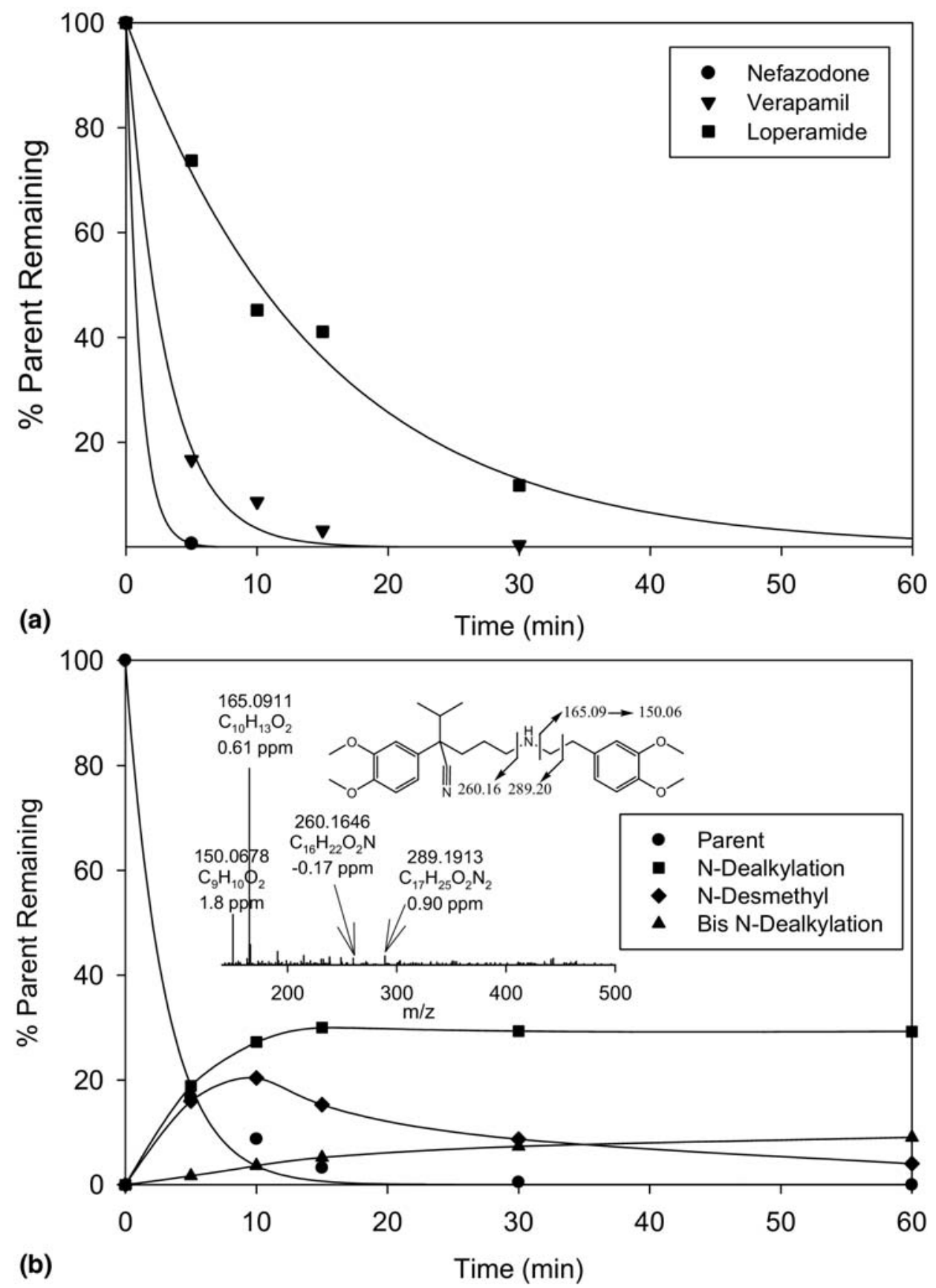

Figure 3. (a) Stability plot for compounds incubated in human microsomes over a time course showing metabolic degradation. (b) Stability plot for verapamil with simultaneous plotting of metabolites detected using the Exactive. Inset in (b) shows HCD spectrum for N-desmethyl metabolite with structure assignment. 
no-metabolism point is arbitrarily set to $100 \%$ parent remaining and all subsequent time points are calculated relative to this sample. As such, standard curves are not used and the instrument response is assumed to be linear across the range of concentrations detected $(\leq 1$ $\mu \mathrm{M})[19,20]$. When using a full-scan approach, no prior method developed is required to determine multiple reaction monitoring (MRM) transitions for the compounds of interest. Post-acquisition data processing requires the monoisotopic mass of the compounds to extract quantitative data.

Microsomal incubations for verapamil were analyzed with the Orbitrap mass analyzer using two scan functions to acquire quantitative and qualitative infor-
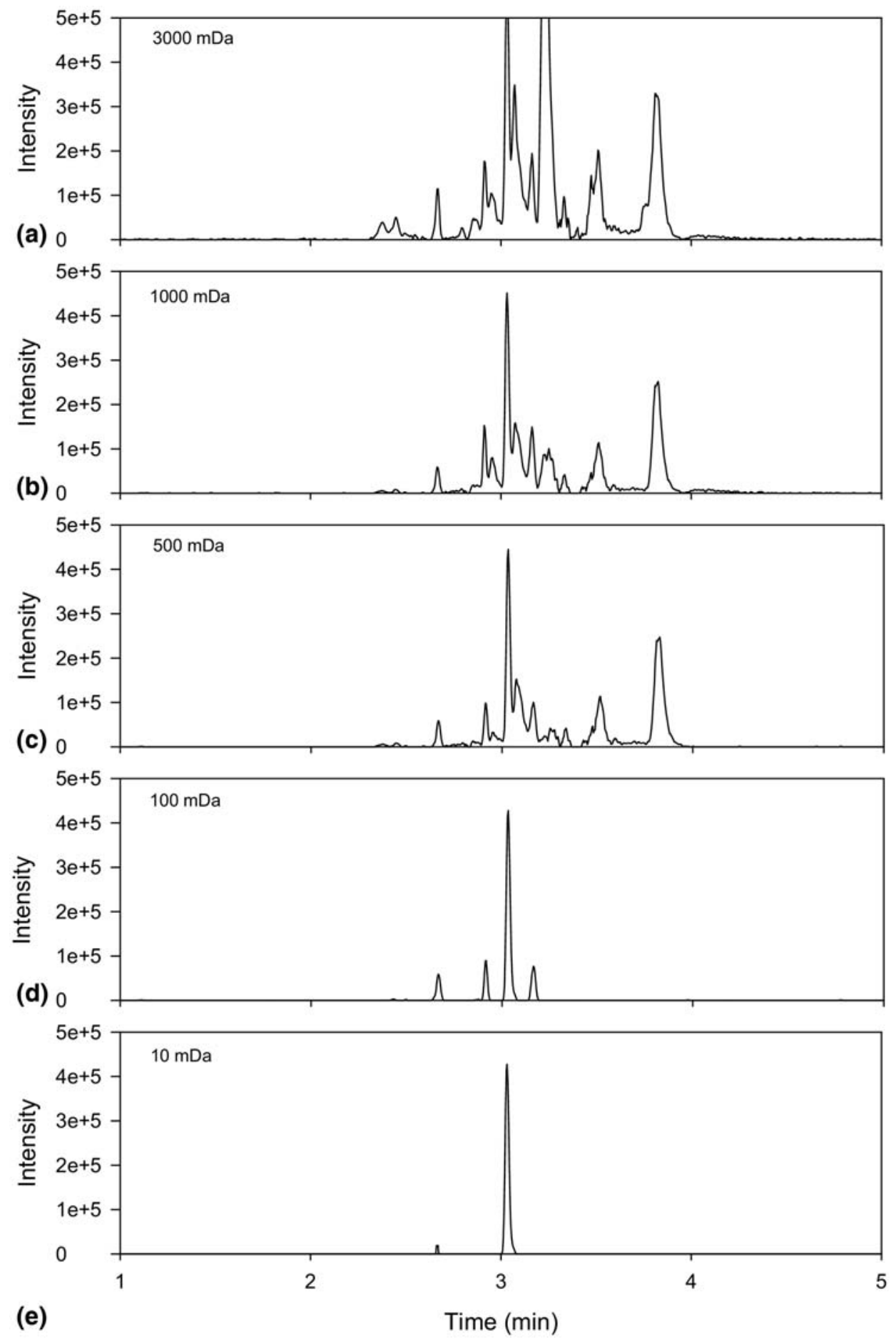

Figure 4. Extracted ion chromatograms with the same data file using decreasing extraction windows (a) $3000 \mathrm{mDa}$, (b) $1000 \mathrm{mDa}$, (c) $500 \mathrm{mDa}$, (d) $100 \mathrm{mDa}$, and (e) $10 \mathrm{mDa}$. Improved selectivity is obtained without loss of sensitivity using high-resolution full-scan data acquisition. 
mation. For each time point the extract ion chromatograms (XICs) for the internal standard and verapamil were generated (data not shown). Selectivity was provided by extracting the $m / z$ values for each component using a 5 ppm window $( \pm 2.5 \mathrm{ppm})$ around the $[\mathrm{M}+$ $\mathrm{H}]^{+}$. At time $=0$ the peak area for $1 \mu \mathrm{M}$ verapamil was about $4.9 \mathrm{e} 6$ and the peak area ratio with the internal standard was 16.35. At time $=30 \mathrm{~min}$, the peak area ratio was 0.076 and, assuming linear response, this represents a concentration of around $4.5 \mathrm{nM}$ verapamil.

When this experiment is repeated for a series of compounds-as is typically done in a discovery screening laboratory to identify metabolically stable versus unstable compounds-a plot such as shown in Figure 3a can be generated. In this case three compounds are plotted, showing low to moderate stability. Providing this type of information to project teams in early discovery work can give the medicinal chemists some information about which chemical series to pursue. However, it would be of greater value if the structural motif(s) leading to lack of metabolic stability could be ascertained from this experiment.

In this case, HCD was acquired at the same time as the MS data. By using knowledge of the fragmentation pattern of the parent compound (Figure $2 \mathrm{~d}$ ), the data can be mined post-acquisition for the presence of metabolites. Equivalent to performing a precursor ion scan in software, fragment ion masses can be extracted from the HCD data to see whether other peaks are present that may correspond to metabolites. In addition, the MS data can be mined with a list of expected metabolite masses. Moreover, fractional mass filtering can be used as another tool to process this high mass resolution dataset $[18,21]$. For this current study, data processing for metabolite identification was done manually and, in the future, software tools will be needed to automate this process if these workflows are to be widely adopted.

Found metabolites can be plotted relative to the parent, with the caveat that their response factors may be different from those of the parent molecule. In a discovery environment this is an acceptable risk, given the benefit derived from knowing sites of metabolism. A plot of parent and metabolites can be generated as shown in Figure $3 \mathrm{~b}$. An example of the HCD for the $\mathrm{N}$-desmethyl metabolite is shown as an inset in Figure $3 b$. Information about location of metabolic soft spots becomes very powerful in the hands of medicinal chemists because they can now modify their synthetic strategy to target the known metabolic soft spots, rather than guessing blindly at what modifications to make.

\section{Quantification of Drugs in Plasma}

Quantitative analysis of target compounds in a complex biological matrix such as plasma requires both selectivity and sensitivity. In the case of triple-quadrupole instruments, selectivity is obtained by using tandem mass spectrometry and sensitivity is provided by the high duty cycle. Full-scan acquisition on a quadrupole mass filter sacrifices both sensitivity and selectivity [7]. For mass analyzers such as time of flight and Orbitrap, sensitivity and selectivity are not mutually exclusive parameters. This is illustrated in Figure 4 for the analysis of a rat plasma sample from a pharmacokinetic study. Data were collected at a resolution setting of 30,000 full-width half-maximum for these plasma samples because it was found that at $R=10,000$ the compound was not completely resolved from endogenous interferences. The chromatograms were generated by extracting $\mathrm{m} / \mathrm{z} 482.1720$ (L-873724) using decreasing extraction window widths around the mass of interest. When a large mass extraction window is used (Figure $4 \mathrm{a}$ and $\mathrm{b}$ ) selectivity is poor and accurate quantification is not possible. As the extraction window is narrowed from $500 \mathrm{mDa}$ (Figure 4c) down to $10 \mathrm{mDa}$ (Figure 4e), the selectivity is improved such that the target compound can be accurately quantified (see the following text). The chromatograms are plotted with the same
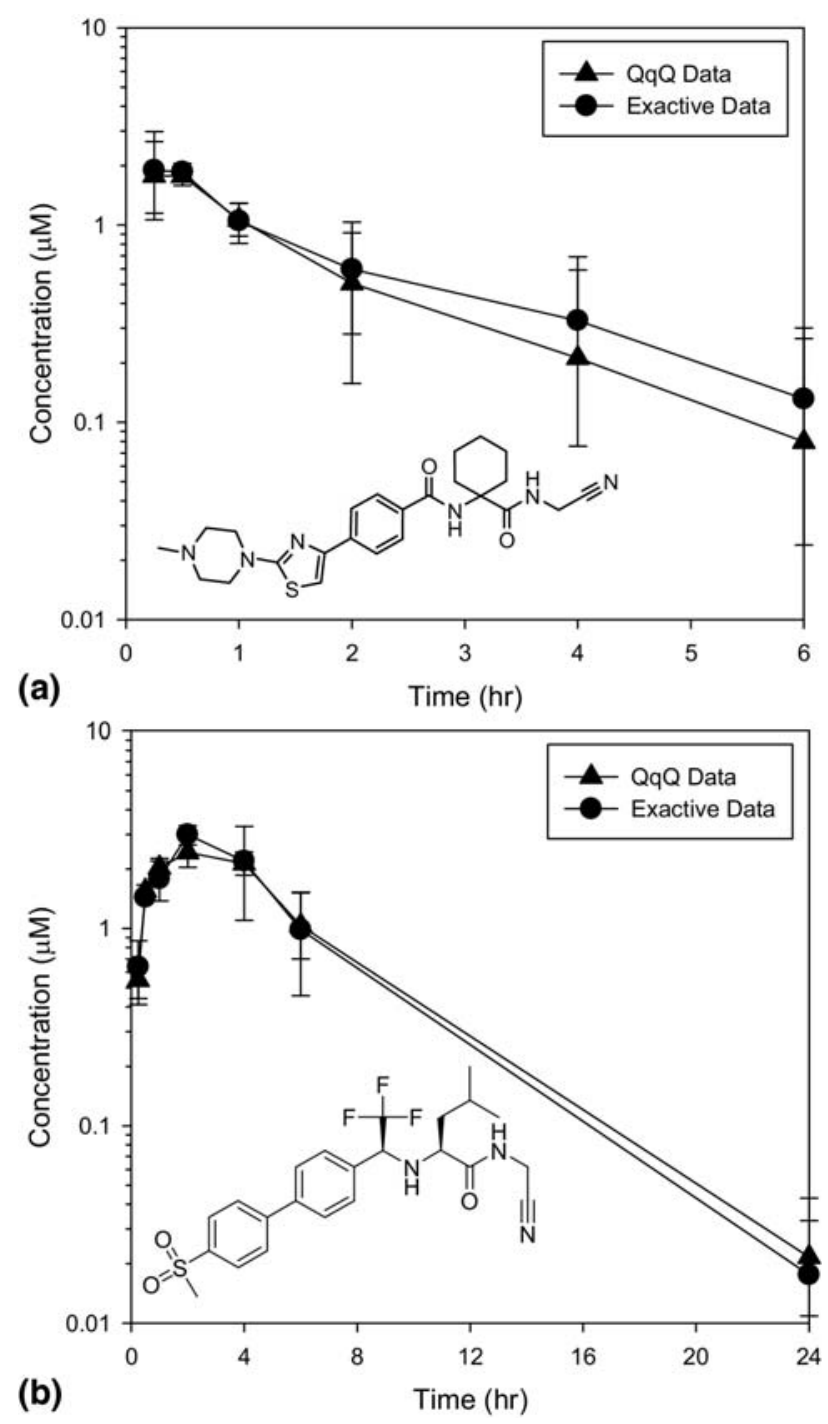

Figure 5. Comparison of triple-quadrupole quantitative analysis with data obtained from the Exactive. (a) L-006235 and (b) L-873724. 
$y$-axis showing that the absolute signal for L-873724 is not lost with decreasing extraction window. It is important to note that mass accuracy must be maintained as the extraction window is decreased; otherwise, the signal would be lost by falling outside the narrow window. It is thus important to have a mass analyzer that maintains mass accuracy over time and over a wide dynamic range of signal; otherwise, accurate quantification will not be possible.

To assess the absolute quantitative capabilities of the system, it was compared with results obtained from triple-quadrupole mass spectrometry. Duplicate standard curves and four levels of quality control samples in triplicate were used to generate the quantitative results of the unknown samples. The resulting pharmacokinetic (PK) curves are plotted in Figure 5. Although the absolute sensitivity of the Orbitrap system is nearly tenfold less than that of the triple quadrupole for the compounds used in this study, this has no effect on the PK curves. As shown in Figure 5, the calculated concentrations for the two compounds in plasma are equivalent within the error of the measurement, independent of mass analyzer. We are therefore confident that we can use a high-resolution full-scan instrument to do absolute quantitative analysis.

Because the data were acquired using full scan, post-acquisition processing for metabolites or other compounds of interest was possible. Figure 6 shows a plot of peak area ratio versus time for L-873724 and a series of metabolites identified in rat plasma. Absolute quantification was not possible in this case because standards for the metabolites were not available. One must thus be careful in interpreting data because it is assumed, in this case, that the metabolites have a response factor similar to that of the parent molecule. Although this may be true in many cases, it is not always so [22, 23]. However, even with these caveats, the data are useful in a discovery environment.

In this case, two metabolites were found to have higher exposure than that of the parent compound. Even though obtaining this information is possible by subsequent analysis of plasma samples for metabolites, this type of experiment is not routinely done because it is time consuming and labor intensive. By using fullscan acquisition, the data are collected up front and, when appropriate, the data file can be processed to look for metabolites. In effect, the metabolite data are collected for "free" and can be mined at a later date. Knowing the sites of metabolism in early discovery gives the chemist the option to block them during lead optimization. Determining this information only in development could result in lengthened timelines and increased costs. In the case of L-873724, the metabolites detected in plasma are shown in Scheme 1. Blocking the two main sites of metabolism on L-873724 led to the discovery of odanacatib, a potent and highly selective cathepsin $\mathrm{K}$ inhibitor [17].

\section{Conclusions}

The use of high-resolution and high mass accuracy data acquisition offers the potential for a fundamental shift

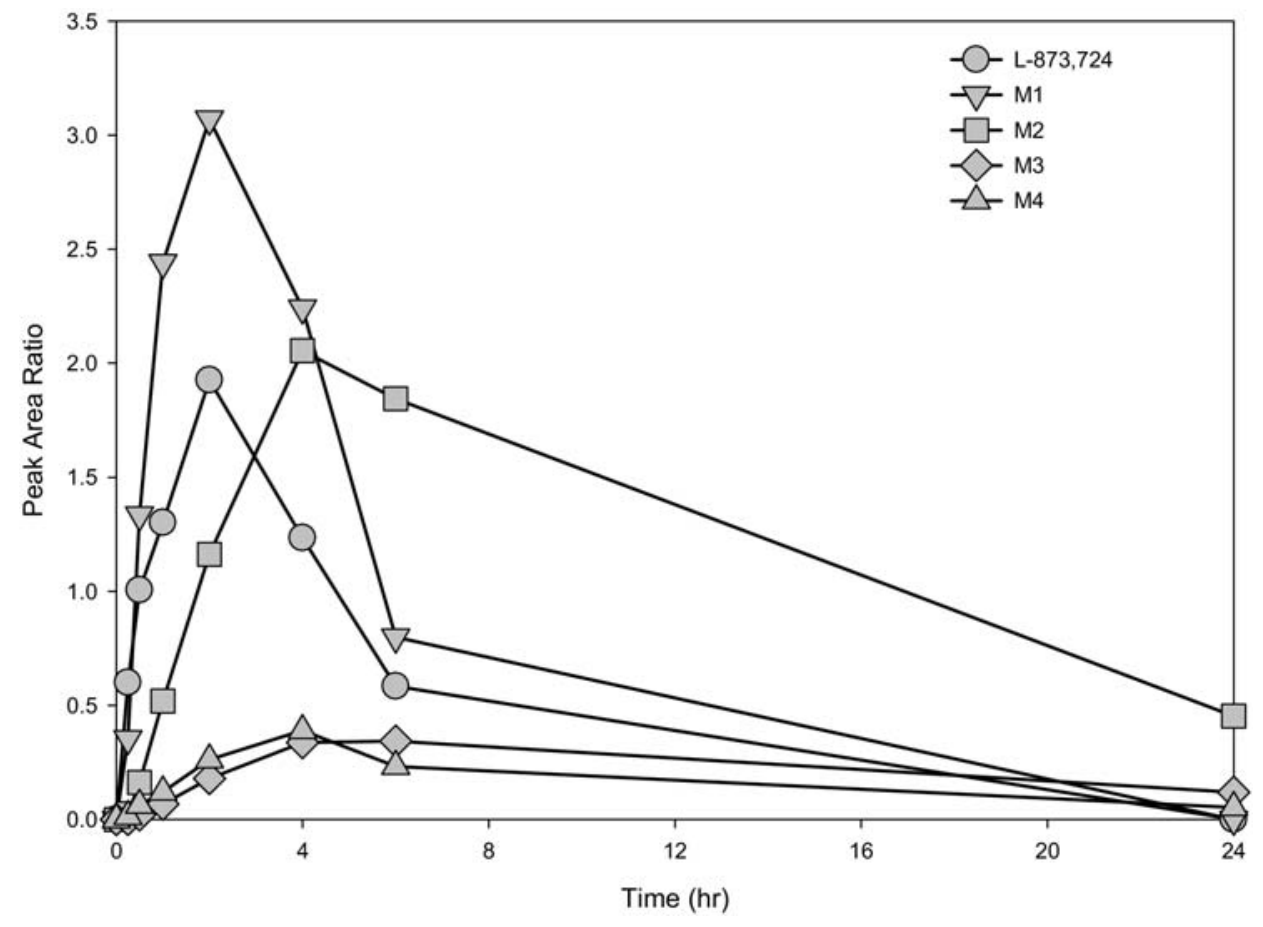

Figure 6. Plot of peak area ratios for L-873724 and metabolites found in rat plasma showing the time course for circulating metabolites. This data were generated post-acquisition by mining of the raw data files. 


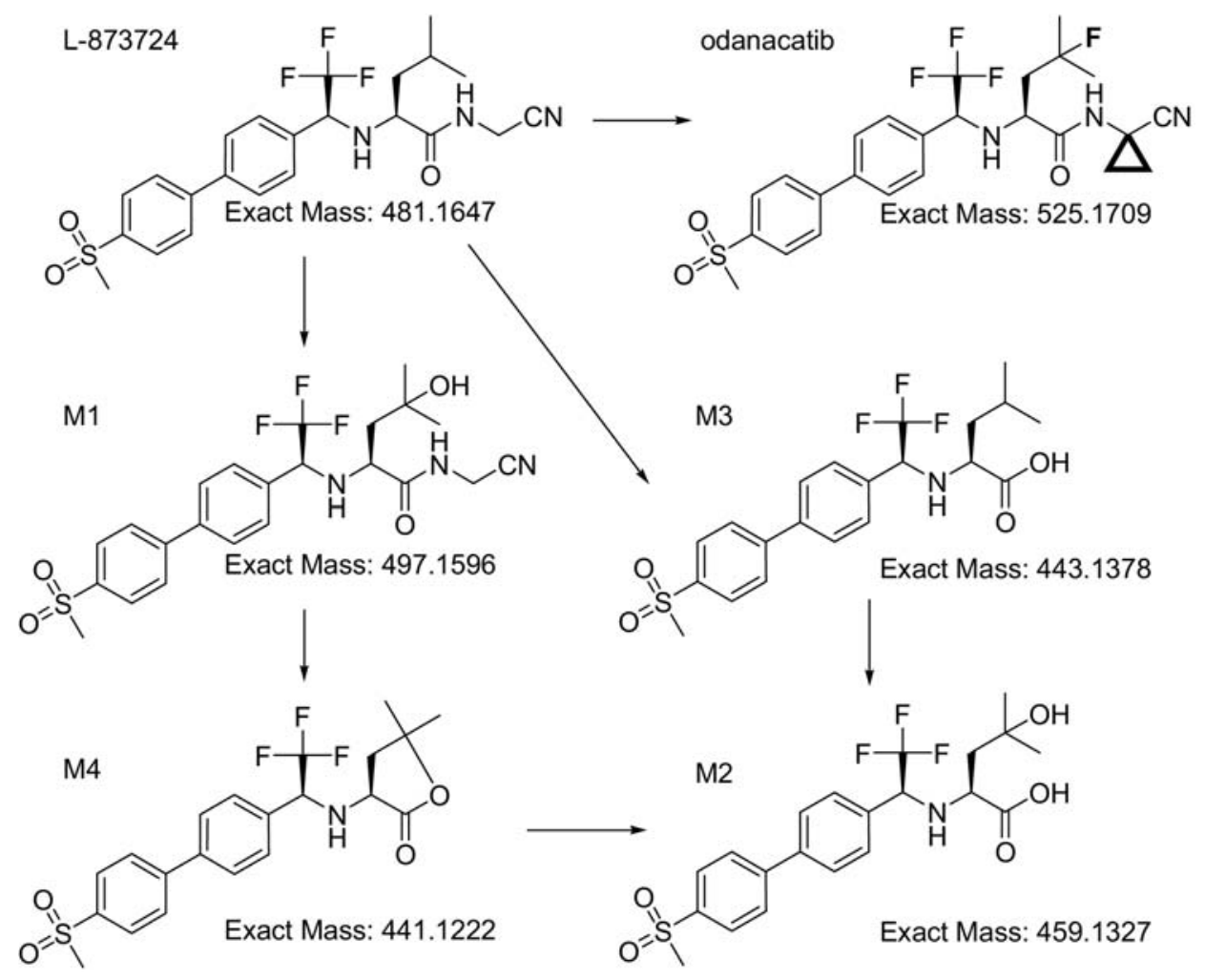

Scheme 1. Metabolite scheme for L-873724 leading to the discovery of odanacatib.

in how routine bioanalysis is carried out in drug discovery. This type of data acquisition-when coupled with sufficient sensitivity, dynamic range, and duty cycle-opens new opportunities for analytical laboratories. The data shown here using the nonhybrid Orbitrap suggest that this type of mass analyzer will be capable of a quantitative-qualitative workflow. It should be emphasized that high resolution $(>20,000)$ and stability of mass accuracy for every peak in every scan are critical to the successful use of full-scan data for quantitative determinations. Full-scan data allow for many possibilities for analysis and post-acquisition processing that are not available when doing targeted analysis. These include, for example, the ability to acquire without the need for SRM method development, dynamic range extension by using isotope data, quantification using HCD data, metabolite identification, and analysis of endogenous compounds. Given the current need for improved success rates in drug discovery and perceived lack of innovation [24], bringing together quantitative and qualitative analyses may provide a capability that opens new doors. For this workflow to be successful, development of software tools that take advantage of full-scan datasets will be required.

\section{References}

1. Gilbert, J. D.; Olah, T. V.; McLoughlin, D. A. High-Performance Liquid Chromatography with Atmospheric Pressure Ionization Tandem Mass Spectrometry: A Tool in Quantitative Bioanalytical Chemistry. Biochem. Biotechnol. Appl. Electrospray Ion. Mass Spectrom. 1996, 619, 330-350.
2. Kola, I.; Landis, J. Can the Pharmaceutical Industry Reduce Attrition Rates? Nat. Rev. Drug Discov. 2004, 3, 711-715.

3. O'Connor, D.; Mortishire-Smith, R.; Morrison, D.; Davies, A.; Dominguez, M. Ultra-Performance Liquid Chromatography Coupled to Time-of-Flight Mass Spectrometry for Robust, High-Throughput Quantitative Analysis of an Automated Metabolic Stability Assay, with Simultaneous Determination of Metabolic Data. Rapid Commun. Mass Spectrom. 2006, 20, 851-857.

4. Zhang, N. Y.; Fountain, S. T.; Bi, H. G.; Rossi, D. T. Quantification and Rapid Metabolite Identification in Drug Discovery Using API Time-ofFlight LC/MS. Anal. Chem. 2000, 72, 800-806.

5. Wieboldt, R.; Campbell, D. A.; Henion, J. Quantitative Liquid Chromatographic Tandem Mass Spectrometric Determination of Orlistat in Plasma with a Quadrupole Ion Trap. J. Chromatogr. B Anal. Technol. Biomed. Life Sci. 1998, 708, 121-129.

6. Rossi, D. T.; Hoffman, K. L.; Janiczek-Dolphin, N.; Bockbrader, H.; Parker, T. D. Tandem-in-Time Mass Spectrometry as a Quantitative Bioanalytical Tool. Anal. Chem. 1997, 69, 4519-4523.

7. King, R. C.; Gundersdorf, R.; Fernandez-Metzler, C. L. Collection of Selected Reaction Monitoring and Full Scan Data on a Time Scale Suitable for Target Compound Quantitative Analysis by Liquid Chromatography/Tandem Mass Spectrometry. Rapid Commun. Mass Spectrom. 2003, 17, 2413-2422.

8. Williamson, L. N.; Zhang, G. D.; Terry, A. V.; Bartlett, M. G. Comparison of Time-of-Flight Mass Spectrometry to Triple Quadrupole Tandem Mass Spectrometry for Quantitative Bioanalysis: Application to Antipsychotics. J. Liquid Chromatogr. Relat. Technol. 2008, 31, 2737-2751.

9. Mauriala, T.; Chauret, N.; Oballa, R.; Nicoll-Griffith, D. A.; Bateman, K. P. A Strategy for Identification of Drug Metabolites from Dried Blood Spots Using Triple-Quadrupole/Linear Ion Trap Hybrid Mass Spectrometry. Rapid Commun. Mass Spectrom. 2005, 19, 1984-1992.

10. Naegele, E.; Fandino, A. S. Simultaneous Determination of Metabolic Stability and Identification of Buspirone Metabolites Using Multiple Column Fast Liquid Chromatography Time-of-Flight Mass Spectrometry. J. Chromatogr. A 2007, 1156, 196-200.

11. Whalen, K. M.; Rogers, K. J.; Cole, M. J.; Janiszewski, J. S. AutoScan: An Automated Workstation for Rapid Determination of Mass and Tandem Mass Spectrometry Conditions for Quantitative Bioanalytical Mass Spectrometry. Rapid Commun. Mass Spectrom. 2000, 14, 2074-2079.

12. Janiszewski, J. S.; Rogers, K. J.; Whalen, K. M.; Cole, M. J.; Liston, T. E.; Duchoslav, E.; Fouda, H. G. A High Capacity LC/MS System for the Bioanalysis of Samples Generated from Plate-Based Metabolic Screening. Anal. Chem. 2001, 73, 1495-1501.

13. Hop, C. E. C. A.; Cole, M. J.; Davidson, R. E.; Duignan, D. B.; Federico, J.; Janiszewski, J. S.; Jenkins, K.; Krueger, S.; Lebowitz, R.; Liston, T. E.; Mitchell, W.; Snyder, M.; Steyn, S. J.; Soglia, J. R.; Taylor, C.; Troutman, 
M. D.; Umland, J.; West, M.; Whalen, K. M.; Zelesky, V.; Zhao, S. X. High Throughput ADME Screening: Practical Considerations, Impact on the Portfolio and Enabler of In Silico ADME Models. Curr. Drug Metab. 2008, 9, 847-853.

14. Makarov, A. Electrostatic Axially Harmonic Orbital Trapping: A HighPerformance Technique of Mass Analysis. Anal. Chem. 2000, 72, 1156-1162.

15. Wieghaus, A.; Makarov, A.; Froehlich, U.; Kellmann, M.; Denisov, E. Lange, O. Development and Applications of a New Benchtop Orbitrap Mass Spectrometer. In Proceedings of the 56th ASMS Conference on Mass Spectrometry and Allied Topics, Denver, CO, 2008.

16. Palmer, J. T.; Bryant, C.; Wang, D. X.; Davis, D. E.; Setti, E. L.; Rydzewski, R. M.; Venkatraman, S.; Tian, Z. Q.; Burrill, L. C.; Mendonca, R. V.; Springman, E.; McCarter, J.; Chung, T.; Cheung, H.; Janc, J. W.; McGrath, M.; Somoza, J. R.; Enriquez, P.; Yu, Z. W.; Strickley, R. M.; Liu, L.; Venuti, M. C.; Percival, M. D.; Falgueyret, J. P.; Prasit, P.; Oballa, R.; Riendeau, D.; Young, R. N.; Wesolowski, G.; Rodan, S. B. Johnson, C.; Kimmel, D. B.; Rodan, G. Design and Synthesis of Tri-Ring P-3 Benzamide-Containing Aminonitriles as Potent, Selective, Orally Effective Inhibitors of Cathepsin K. J. Med. Chem. 2005, 48, 7520-7534.

17. Gauthier, J. Y.; Chauret, N.; Cromlish, W.; Desmarais, S.; Duong, L. T.; Falgueyret, J. P.; Kimmel, D. B.; Lamontagne, S.; Leger, S. LeRiche, T.: Li, C. S.; Masse, F.; McKay, D. J.; Nicoll-Griffith, D. A. Oballa, R. A.; Palmer, J. T.; Percival, M. D.; Riendeau, D.; Robichaud, J.; Rodan, G. A.; Rodan, S. B.; Seto, C.; Therien, M.; Truong, V. L.; Venuti, M. C.; Wesolowski, G.; Young, R. N.; Zamboni, R.; Black, W. C. The Discovery of Odanacatib (MK-0822), a Selective Inhibitor of Cathepsin K. Bioorg. Med. Chem. Lett. 2008, 18, 923-928.

18. Wrona, M.; Mauriala, T.; Bateman, K. P.; Mortishire-Smith, R. J.; O'Connor, D. "All-in-One" Analysis for Metabolite Identification Using
Liquid Chromatography/Hybrid Quadrupole Time-of-Flight Mass Spectrometry with Collision Energy Switching. Rapid Commun. Mass Spectrom. 2005, 19, 2597-2602.

19. Di, L.; Kerns, E. H.; Gao, N.; Li, S. Q.; Huang, Y. P.; Bourassa, J. L.; Huryn, D. M. Experimental Design on Single-Time-Point HighThroughput Microsomal Stability Assay. J. Pharm. Sci. 2004, 93, 15371544 .

20. Drexler, D. M.; Belcastro, J. V.; Dickinson, K. E.; Edinger, K. J.; Hnatyshyn, S. Y.; Josephs, J. L.; Langish, R. A.; McNaney, C. A.; Santone, K. S.; Shipkova, P. A.; Tymiak, A. A.; Zvyaga, T. A.; Sanders, M. An Automated High Throughput Liquid Chromatography-Mass Spectrometry Process to Assess the Metabolic Stability of Drug Candidates. Assay Drug Dev. Technol. 2007, 5, 247-264.

21. Bateman, K. P.; Castro-Perez, J.; Wrona, M.; Shockcor, J. P.; Yu, K. Oballa, R.; Nicoll-Griffith, D. A. MSE with Mass Defect Filtering for In Vitro and In Vivo Metabolite Identification. Rapid Commun. Mass Spectrom. 2007, 21, 1485-1496.

22. Valaskovic, G. A.; Utley, L. Lee, M. S. Wu, J. T. Ultra-Low Flow Nanospray for the Normalization of Conventional Liquid Chromatography/Mass Spectrometry through Equimolar Response: Standard-Free Quantitative Estimation of Metabolite Levels in Drug Discovery. Rapid Commun. Mass Spectrom. 2006, 20, 1087-1096.

23. Tiller, P. R.; Yu, S.; Bateman, K. P.; Castro-Perez, J.; Mcintosh, I. S.; Kuo, Y. S.; Baillie, T. A. Fractional Mass Filtering as a Means to Assess Circulating Metabolites in Early Human Clinical Studies. Rapid Commun. Mass Spectrom. 2008, 22, 3510-3516.

24. Kola, I. The State of Innovation in Drug Development. Clin. Pharmacol. Ther. 2008, 83, 227-230 